\title{
Multibunch beam extraction using the strip-line kicker at the KEK Accelerator Test Facility
}

\author{
T. Naito, ${ }^{*}$ S. Araki, H. Hayano, K. Kubo, S. Kuroda, N. Terunuma, T. Okugi, and J. Urakawa \\ KEK, Tsukuba, Japan
}

(Received 27 October 2010; published 18 May 2011)

\begin{abstract}
The International Linear Collider (ILC) damping ring (DR) injection and extraction kickers have a very special role: the bunch spacing 189-480 ns is compressed to 3-9 ns when injected into the DR and then decompressed to 189-480 ns when leaving the DR. The kickers act as a bunch-by-bunch beam manipulator to compress and decompress the bunch spacing into/from the DR. They require a fast rise/ fall time (3-9 ns) and a high repetition rate (6-2 MHz). Among the candidate technologies, the multiple strip-line kicker system is the most likely to realize the specifications for the ILC reference design. A beam extraction experiment with a prototype strip-line kicker has been carried out at the KEK Accelerator Test Facility (ATF). The kicker is composed of two units of 60-cm-long strip-line electrodes. The multibunch beam (30 bunches spaced at $5.6 \mathrm{~ns}$ ) stored in the DR was extracted successfully with a bunch spacing of $308 \mathrm{~ns}$. The measured stability of the kick angle was $3.5 \times 10^{-4}$. Some, but not all, parameters of the tested kicker meet the ILC-DR injection/extraction kicker requirements.
\end{abstract}

DOI: 10.1103/PhysRevSTAB.14.051002

PACS numbers: 29.20.-c, 29.27.Ac, 42.79.Fm

\section{INTRODUCTION}

The International Linear Collider (ILC) is an electronpositron collider with a $200-500 \mathrm{GeV}$ center of mass energy. The design work and hardware developments are done in the framework of a worldwide collaboration. The damping rings (DRs) for the electron and positron beam play a significant role in realizing the high luminosity at the collision point. The beam of the ILC is a long bunch train spanning a length of over $200 \mathrm{~km}$ long. It consists of 1320-5120 bunches with a bunch spacing of $189-480 \mathrm{~ns}$ in the linacs. The bunch spacing should be compressed to fit the actual size of the DR. The circumference of the DR is $6.7 \mathrm{~km}$ and the bunch spacing is 3-9 $\mathrm{ns}$ in the base-line design [1]. Some of the parameters are shown as ranges in the design. The ILC design specifies that each subsystem support a range of beam parameters. The resulting flexibility in operating parameters improves operation: if problems are identified in one area, they can be compensated for in another [1]. The kicker parameters are also shown as ranges to fit the beam parameters.

The injection/extraction kickers act as a bunch-by-bunch beam manipulator to compress and decompress the bunch spacing into/from the DR. The kick field is designed to manipulate only the deflecting bunch without affecting the next/previous bunches traveling after/before it at 3 (to 9) ns intervals. The kicker requires a high repetition frequency of 6 (to 2) $\mathrm{MHz}$ and a very fast rise/fall time of 3 (to 9) ns for the kick field. Most of the major accelerator complexes

\footnotetext{
*takashi.naito@kek.jp
}

Published by the American Physical Society under the terms of the Creative Commons Attribution 3.0 License. Further distribution of this work must maintain attribution to the author(s) and the published article's title, journal citation, and DOI. in operation around the world use a pulsed magnet system consisting of a ferrite pulsed magnet powered by a thyratron switching pulsed power supply. The typical rise time is $60 \mathrm{~ns}$ and the repetition rate is a few hundred hertz [2]. The cutoff frequency of the pulsed magnet limits the rise/fall time of the kick field. There is no feasible way to realize the ILC parameters by extending the conventional kicker technology. Among the candidate technologies, a system using multiple strip-line kickers appears to be the most likely to realize the parameters. In work at the accelerator test facility damping ring (ATF DR) in the High Energy Accelerator Research Organization, Naito et al. have already tested a single strip-line kicker using a high-voltage semiconductor pulse source and measured the rise/fall time of the beam kick field [3,4]. Grishanov et al., meanwhile, have already proposed a strip-line kicker using a single unit for TESLA [5], and Alesini et al. have developed a tapered strip line for the injection of DAФNE [6]. Their designs and results provide useful information for our own work.

The multiunit design and the burst operation are required to confirm the performance of the strip-line kicker system for the ILC. The beam extraction experiment from the ATF DR to the extraction line with a prototype stripline kicker was proposed [7]. The characteristics of the extracted beam can be measured in this experiment using the beam position monitors at the extraction line.

\section{STRIP-LINE KICKER FOR ILC DR}

Figure 1 shows a schematic of the strip-line kicker system for the ILC DR. Table I shows the parameters of the ILC-DR kicker and the parameters achieved at ATF. The strip-line kicker system consists of 15 or more stripline kicker units. Each unit consists of a trigger delay, a pair of $5 \mathrm{kV}$ pulse generators with negative and positive 


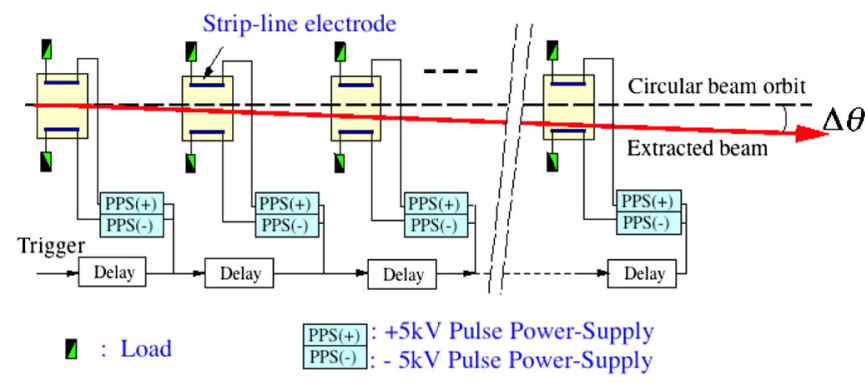

FIG. 1. Schematic layout of the strip-line kicker for the ILC DR: The kicker consists of 15 or more strip-line units. The length of the strip line is limited to realize the rise/fall time and the kick angle of each unit is small. Multiunit is required to realize the specified kick angle.

voltages, and a pair of parallel plates of strip-line electrodes. Each unit in the system has a strip-line length $L$, a distance $d$ between the electrodes, and an applied pulse voltage of $V$ for the two electrodes. The kick angle of a single unit, $\Delta \theta$, is given as

$$
\Delta \theta=2 g \frac{e V}{E} \frac{L}{d}
$$

where $E$ is the beam energy and $g$ is the geometry factor determined by the shape of the electrode. The geometry factor $g_{\perp}$ is given as

$$
g_{\perp}=\tanh \left(\frac{\pi w}{2 d}\right)
$$

where $w$ is the strip-line width. When the appropriate pulse timing is set for each strip-line electrode, the total kick field is the sum of all single-unit kick fields. In the case of $E=5 \mathrm{GeV}, L=30 \mathrm{~cm}, d=30 \mathrm{~mm}$, and $g=1$, we need a pulse voltage of $150 \mathrm{kV}$ to provide a kick angle of $0.6 \mathrm{mrad}$. When using $10 \mathrm{kV}(+/-5 \mathrm{kV})$ pulse power supplies, the system needs 15 or more strip-line kicker units.

The length of the strip-line electrode limits the rise/fall time of the kick field. The electric and magnetic fields of the kicker pulse are transmitted at the speed of light in the strip-line electrode, and a beam travels at the same speed in the opposite direction. The beam is subjected to electric and magnetic fields with a rise time of $2 \mathrm{~L} / \mathrm{c}$. The kick field the beam encounters is expressed by integrating the voltage waveform $[V(t)]$ over $\tau_{g}=2 \mathrm{~L} / \mathrm{c}$, where $\tau_{g}$ is the "kick growth time" [7]. When a pulse is applied to the strip line, the kick field is

$$
\Delta \theta(t)=2 g \frac{e}{E} \frac{L}{d} \int_{t}^{t+\tau_{g}} V(t) d t
$$

Figures 2(a) and 2(b) show a measured waveform of the kicker pulse voltage (see Sec. III C) and the estimated kick field when the kicker pulse is applied to a 60-cm-long strip line. The rise time of the kick field is less than $5 \mathrm{~ns}$. To realize a rise time of less than $3 \mathrm{~ns}$, the strip line has to be shortened to a length of $30 \mathrm{~cm}$, which in this case reduces the kick field by half. Our group proposed and tested a method to improve the rise/fall time of the kick field in an earlier study [4]. If a small kick field with negative direction is applied to the timing of the rising/falling edge of the main kick field, the superimposed kick field is steeper than the main kick field. This method can be also used to cancel the residual kick field coming from the reflection of the kicker pulse. Figure 2(c) shows the peak area of Fig. 2(b). There is a continuous change in the kick angle as a function of time, and the gradient does not fall to zero for any more than an instant for a period of less than 200 ps. It means that the timing jitter between the beam and the kicker pulse causes kick angle jitter. The minimum change in kick angle over any 200 ps period is $0.3 \mu \mathrm{rad}$.

TABLE I. Parameters of the ILC-DR kicker and parameters achieved at ATF.

\begin{tabular}{lll}
\hline \hline & ILC design/requirements & ATF achieved \\
\hline Beam energy & $5 \mathrm{GeV}$ & $1.3 \mathrm{GeV}$ \\
Bunch space in DR & $3-9 \mathrm{~ns}$ & $5.6 \mathrm{~ns}$ \\
Bunch space in extraction line & $189-480 \mathrm{~ns}$ & $308 \mathrm{~ns}$ \\
Kick angle & $0.6 \mathrm{mrad}$ & $3 \mathrm{mrad}$ \\
Number of kicker units & $15 \mathrm{or} \mathrm{more}$ & 2 \\
Repetition rate in a burst & $6-2 \mathrm{MHz}$ & $3.3 \mathrm{MHz}$ \\
Number of macropulses & $1320 / 2625 / 5120$ & 30 \\
Repetition rate of the macropulse & $5 \mathrm{~Hz}$ & $0.5 \mathrm{~Hz}$ \\
Rise and fall time & $<3-9 \mathrm{~ns}$ & $<5.0 \mathrm{~ns}$ (3 ns) \\
Peak kick stability & $0.07 \%$ & $0.034 \%$ (@ single bunch) \\
Amplitude change in a burst & $0.07 \%{ }^{\mathrm{c}}$ & $1 \%{ }^{\mathrm{d}}$ \\
\hline \hline
\end{tabular}

${ }^{a}$ Number of kicker units depends on the design of the strip line.

${ }^{\mathrm{b}}$ Time for amplitude reduction of factor 0.001 from the peak.

${ }^{\mathrm{c}}$ This will be loosened significantly by feedback/feed forward.

${ }^{\mathrm{d}}$ Excluding the first 18 pulses. 

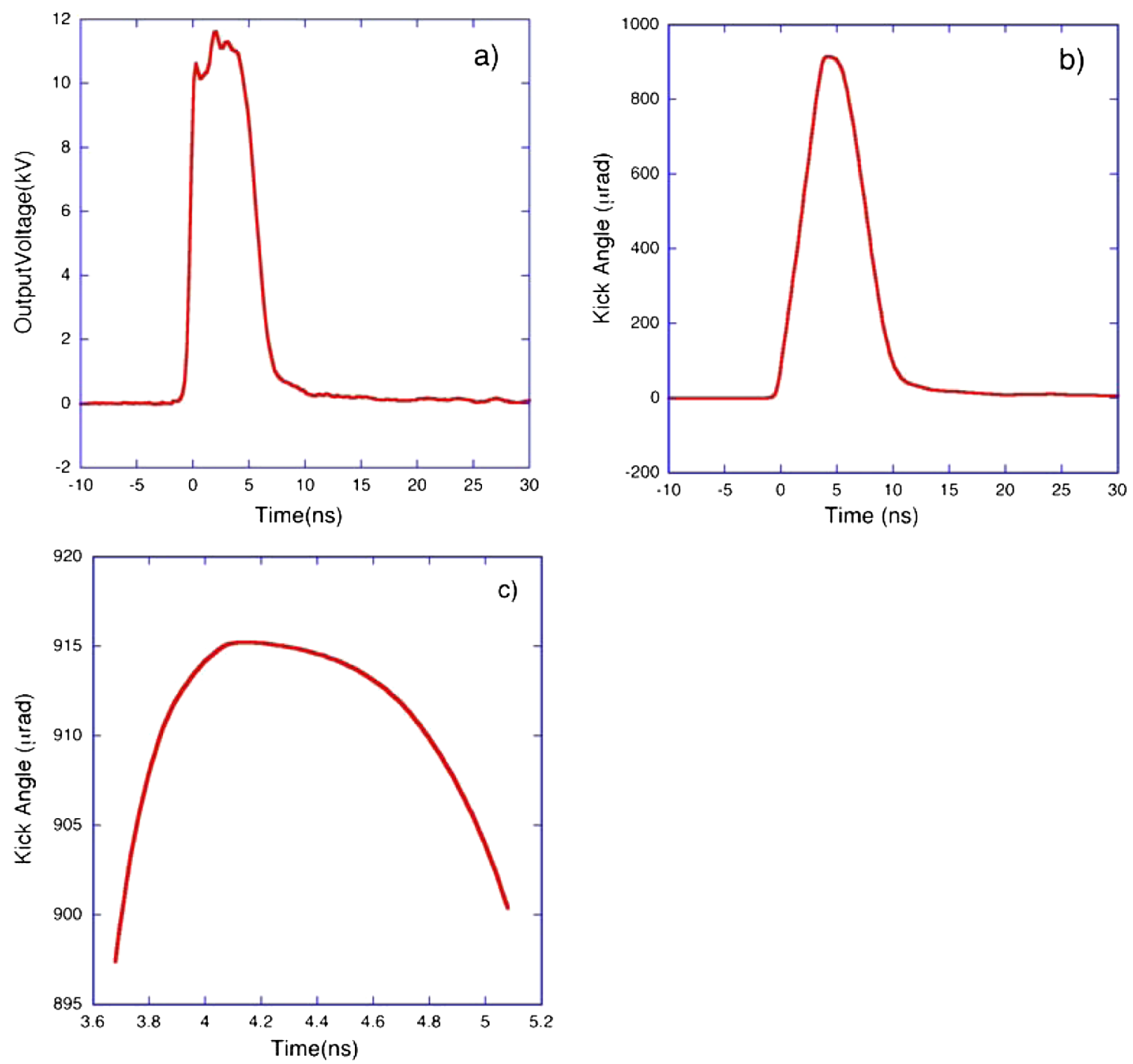

FIG. 2. (a) The waveform of the kicker pulse (positive polarity); (b) the estimated kick field as a function of time when the kicker pulse is applied to a 60-cm-long strip line; (c) the expanded peak area of the estimated kick field: There is a continuous change in the kick angle as a function of time, and the gradient does not fall to zero for any more than an instant for a period of less than 200 ps.

\section{BEAM EXTRACTION EXPERIMENT AT THE ATF}

The main parameters of the ATF DR are a beam energy of $1.3 \mathrm{GeV}$, circumference of $138 \mathrm{~m}$, and revolution time of $462 \mathrm{~ns}$ [8]. The stored beam consists of three trains with a spacing of $103.6 \mathrm{~ns}$. One train is injected in one cycle. Each train consists of ten bunches with a bunch spacing of $5.6 \mathrm{~ns}$. There are a total of 30 bunches in the DR. The beam injection rate is $1.5 \mathrm{~Hz}$ under normal operating conditions and the typical intensity is $10^{10}$ electrons/bunch. The stripline kicker kicks out bunch by bunch from the last bunch of each train at an interval of $308 \mathrm{~ns}$. The interval is changed to $302.4 \mathrm{~ns}$ for every third pulse, to extract the next bunch in the train. The strip-line kicker needs to have a rise time of less than $5.6 \mathrm{~ns}$, a burst of 3.24 (3.30) $\mathrm{MHz}$ over 30 pulses, and a repetition rate of $0.5 \mathrm{~Hz}$. Figure 3 shows the bunch structure in the DR and the order of the bunches at the extraction line.

The most difficult parameter to satisfy for beam extraction when switching from the existing pulsed magnet to the strip-line kicker is the kick angle. The pulsed magnet of the present system is $60 \mathrm{~cm}$ in length and produces a kick angle of $4.6 \mathrm{mrad}$ in the horizontal direction. There is no space to install the strip-line electrodes necessary to attain the same kick angle of the pulsed magnet. The strip-line kicker can produce a kick angle of up to $3 \mathrm{mrad}$ with two units installed in the $1.5 \mathrm{~m}$ available space. The beam cannot be extracted solely via the deflection of the strip-line kicker.

We designed a process that combines a local bump orbit, beam deflection with the strip-line kicker, and an auxiliary septum. Figure 4 shows beam extraction orbits the system with the pulsed magnet and the system with the strip-line 


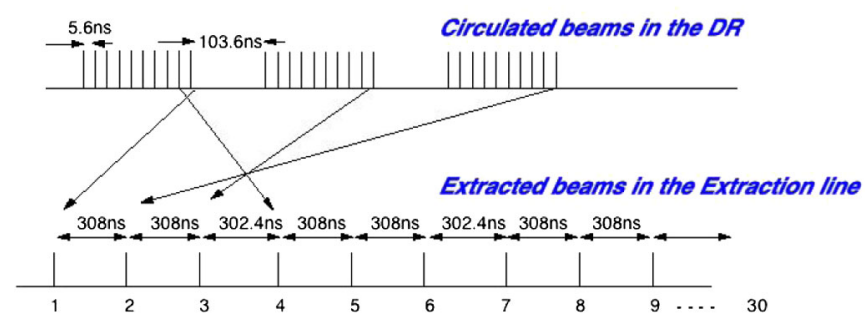

FIG. 3. Bunch structure in the DR and in the extraction line: The bunch stored in the DR is three trains and each train consists of ten bunches with $5.6 \mathrm{~ns}$ bunch spacing. The beam is extracted bunch by bunch from the last bunch of each train with $308 \mathrm{~ns}$ spacing. The bunch spacing changes to 302.4 ns after every third bunch is extracted.

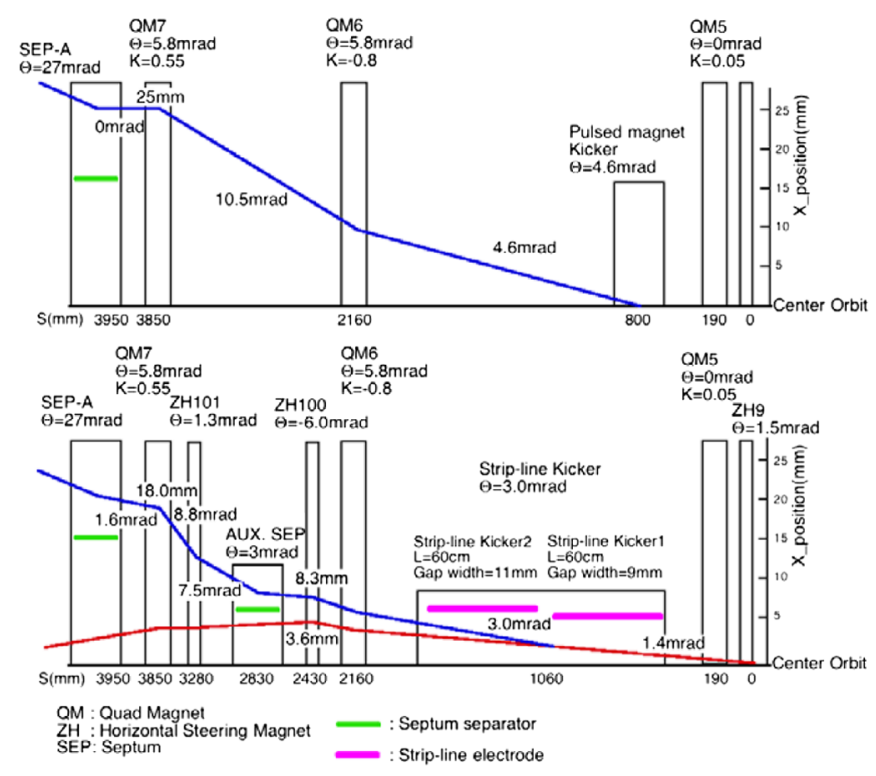

FIG. 4. Comparison of the beam extraction orbits (blue lines) in two systems: one with a pulsed magnet (upper) and one with a strip line (lower). The pulsed magnet kicker produces a $4.6 \mathrm{mrad}$ kick angle and $25 \mathrm{~mm}$ displacement at the entrance of septum A. The strip-line kicker produces a $3.0 \mathrm{mrad}$ kick angle. The stored orbit moves outside of the DR (local bump orbit, shown as red line), whereupon the strip-line kicker kicks the beam. The auxiliary septum expands the small orbit difference. The extracted beam has an $18 \mathrm{~mm}$ displacement and a $1.6 \mathrm{mrad}$ angle at the entrance of septum A.

kicker. The beam is extracted in the horizontal direction in both systems. With the pulsed magnet kicker, the kicker produces a $4.6 \mathrm{mrad}$ kick angle to the beam. The extracted beam has a $25 \mathrm{~mm}$ displacement and $0 \mathrm{mrad}$ angle at the entrance of septum A. With the strip-line kicker, the local bump orbit moves the stored orbit to the outside of the DR, whereupon the strip-line kicker kicks the beam $3 \mathrm{mrad}$. The auxiliary septum expands the small orbit difference of the kicked beam. The extracted beam has an $18 \mathrm{~mm}$ displacement and a $1.6 \mathrm{mrad}$ angle at the entrance of septum A. Figure 5 shows the time sequence of the beam

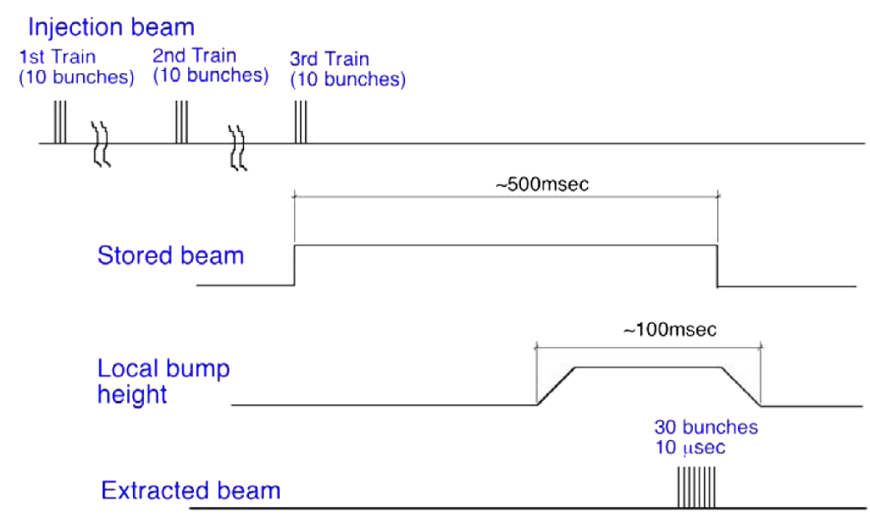

FIG. 5. Time sequence of the beam injection and extraction: (1) the three trains of the beam are injected to the DR; (2) the local bump orbit is excited gradually after the beam is damped; (3) the beam is kicked bunch by bunch at the end of the flattop of the bump orbit; and (4) the bump orbit returns to zero.

extraction in the system with the strip-line kicker. The process proceeds as follows: (1) the three trains of the beam are injected to the DR; (2) the local bump orbit is excited gradually after the beam is damped; (3) the beam is kicked bunch by bunch at the end of the flattop of the bump orbit; and (4) the bump orbit returns to zero. The local bump must be excited after the stored beam is damped, in order to keep the aperture wide enough for the injection beam.

\section{A. Local bump}

The peak of the local bump is set at the ZH100 steering magnet, a component newly installed for the local bump (see Fig. 4). The auxiliary septum is positioned immediately downstream of the ZH100 steering magnet. The extracted beam passes through the inside of the auxiliary septum and changes the direction. Seven steering magnets are used to generate the local bump, which is set to zero for the horizontal position and the dispersion function at the outside of the bump. The height of the local bump can be set arbitrarily. The shape of the local bump is maintained by synchronizing the currents of all of the steering magnets in the local bump.

\section{B. Auxiliary septum}

The design of the auxiliary septum is based on the PEP-II septum [9]. Table II shows the main parameters of the septum. The thickness of the septum separator is

TABLE II. Parameters of the auxiliary septum.

\begin{tabular}{ll}
\hline \hline Magnet length & $60 \mathrm{~cm}$ \\
Magnet gap & $10 \mathrm{~mm}$ \\
Bending field & $0.06 \mathrm{~T}$ \\
Magnet current & $600 \mathrm{~A}$ \\
Separator width & $1.6 \mathrm{~mm}$ \\
\hline \hline
\end{tabular}


only $1.6 \mathrm{~mm}$. A hollow conductor cannot be used as a thin separator, as the cooling channels need the space inside the separator. The cooling channels are made of stainless steel and welded onto the upper and lower sides of the separator. The dimensions of the septum were designed with OPERA software [10]. The auxiliary septum is installed in the vacuum chamber and the power cables and cooling channels are connected through the ceramic feedthrough. The magnetic field has been checked at levels of up to $600 \mathrm{~A}$ without any heat problems. The leakage flux is less than $1 \%$ of the main flux and can be canceled out by the auxiliary coil of the septum.

\section{Strip-line kicker}

Figure 6 shows a schematic layout of the strip-line kicker for the ATF. The length of the strip line is $60-\mathrm{cm}-$ long with the gaps of 9 and $11 \mathrm{~mm}$. The short rise time required of the kick field limits the length. The gaps are determined by the aperture for the injection beam and by the kick angle. The extracted beam passes through $4 \mathrm{~mm}$ off center at the end of the downstream strip line, hence a

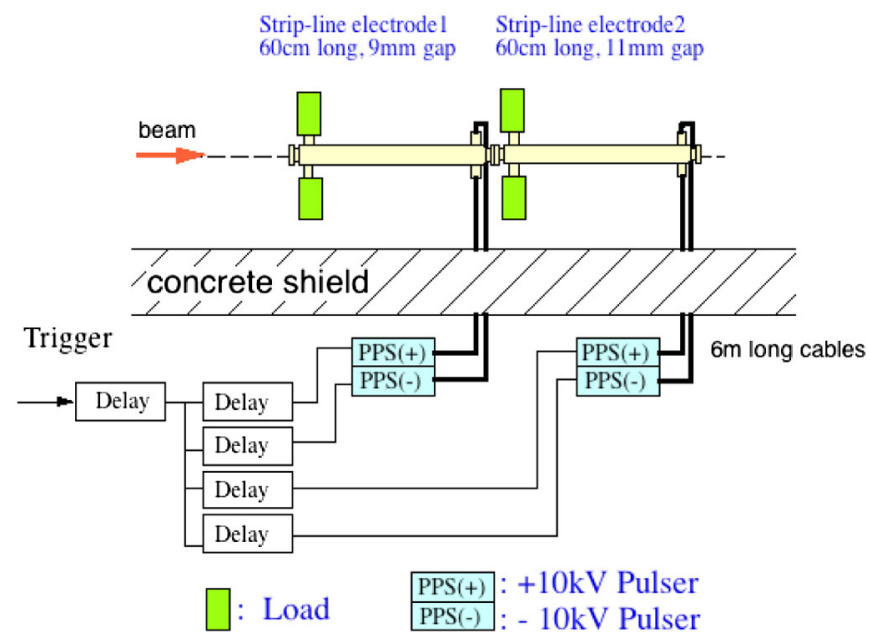

FIG. 6. Schematic layout of the strip-line kicker system for ATF.

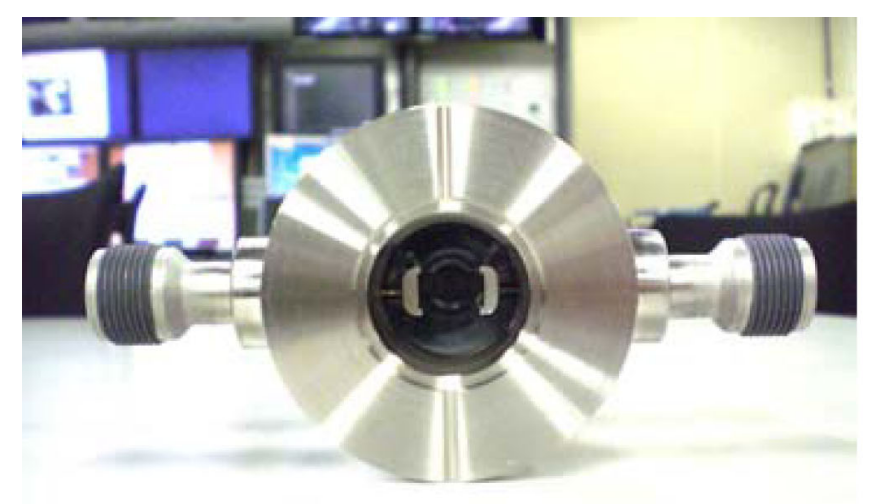

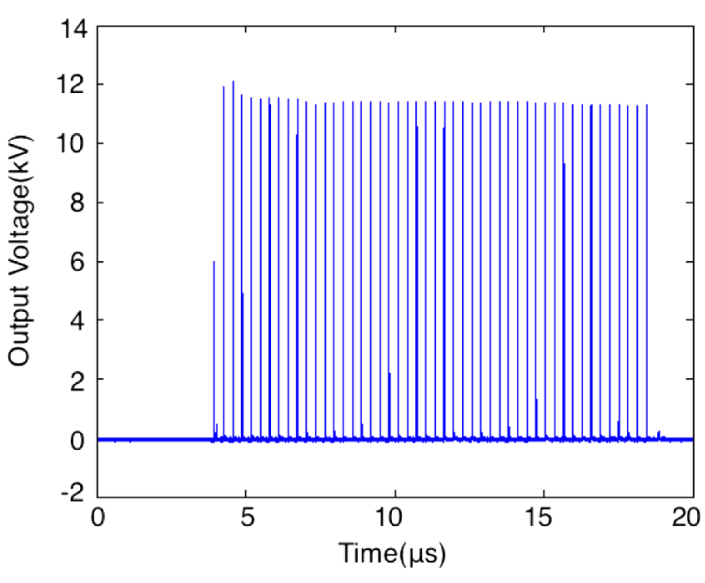

FIG. 8. Burst pulse waveforms of FPG10-3000KN.

wider gap is required for the downstream strip line. The pulsers are located outside of the shielding and the highvoltage pulses are transmitted by 6-m-long coaxial cables. The trigger timing signals for two pairs of pulsers are independently controlled by four delay circuits.

Figure 7 shows a side view of the strip-line electrode and the simulated electric and magnetic field. The impedance of the electrode is maintained at $50 \Omega$. POISSON software was used to design an electrode shape [11] that would generate a peak electric field small enough to avoid discharge. A high voltage version of the $N$ connector type commercially available feedthroughs are installed as the input/output connectors.

Two pairs of FPG10-3000KN pulsers (fabricated by FID Co., Ltd. [12]) are used for the pulse source. The main parameters of the pulsers are a peak amplitude of $10 \mathrm{kV}$, rise time of $1.5 \mathrm{~ns}$, repetition rate of $3.3 \mathrm{MHz}$ (burst), and bursts of up to 60 pulses at $1 \mathrm{~Hz}$. Figure 2(a) shows a single pulse waveform and Fig. 8 shows the burst pulse waveform from the 1st to 48th pulse. The last 30 pulses are used for the beam kick because the amplitude and the timing are stable. (Details on this are discussed in Sec. IIIE.) If we examine the waveform of the burst pulse, we see that the

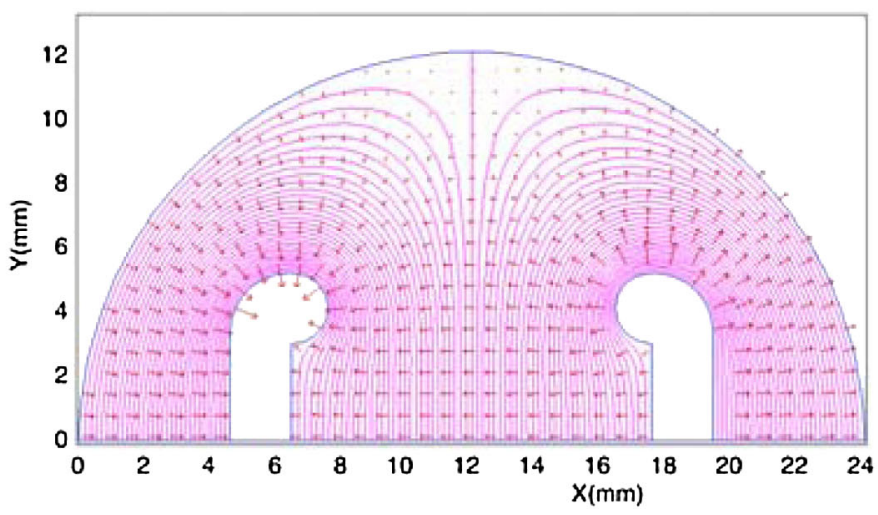

FIG. 7. Photograph of the strip-line electrode and diagram of the simulated electric and magnetic field. 
first pulse has insufficient amplitude and the following pulses have droop. The amplitude change from 18th to the last pulse is about $1 \%$. Figure 2(b) shows the time profile of the kick field. The kick field has a rise time of less than $5 \mathrm{~ns}$ but no flattop. The kick angles are $1.8 \mathrm{mrad}$ for the $9 \mathrm{~mm}$ gap width strip line and $1.5 \mathrm{mrad}$ for the $11 \mathrm{~mm}$ gap width strip line, without accounting for cable loss or the reflection coming from the mismatch at the strip line. If we account for these effects, the angles will presumably be about $10 \%$ lower than the estimated values. When two 60 -cm-long strip lines with $\pm 10 \mathrm{kV}$ pulsers are used, the resulting total kick angle is $3 \mathrm{mrad}$.

\section{The output pulse timing drift of the pulser}

The temperature change of FPG10-3000KN pulsers induces a 400-600 ps timing drift in the output timing per day, which is characteristic of the pulser. This timing drift has to be stabilized if stable kicker operation is to be attained. We tested the timing feedback system using an oscilloscope and fine delays. The readout value for the output timing on the oscilloscope is fed back to the fine delay setting input via computer control. The green line in Fig. 9 plots the value set for the fine delay, which changes by over 400 ps per day. The red line shows the output timing under the feedback condition, which is kept in the range of $200 \mathrm{ps}$.

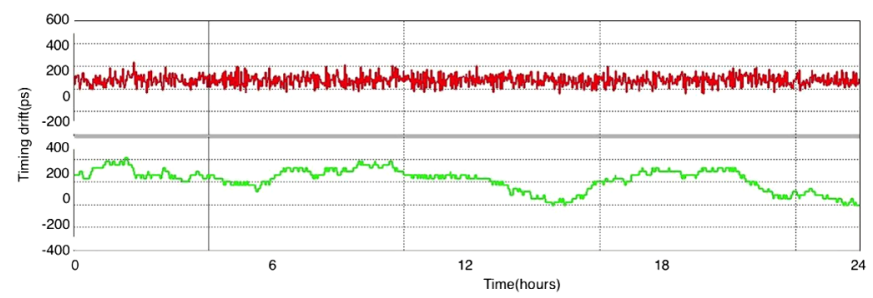

FIG. 9. Output timing feedback: the fine delay setting (green) and the output timing (red).

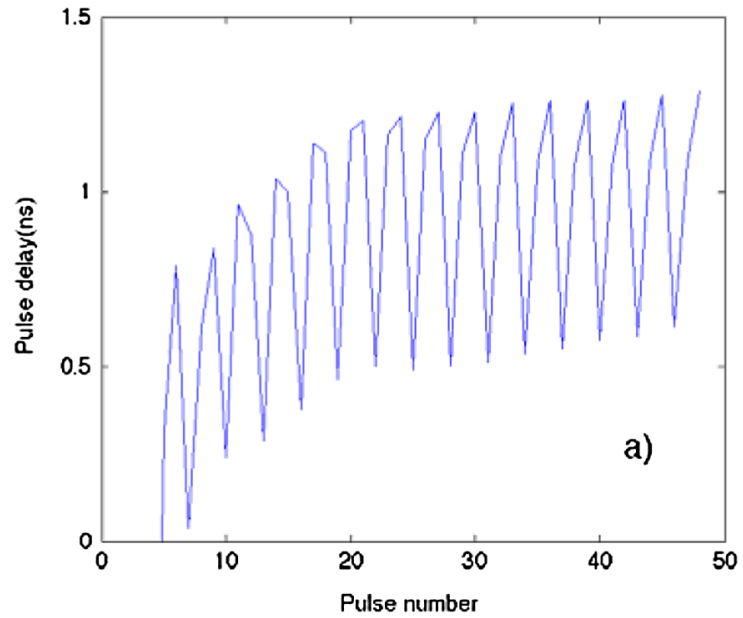

\section{E. Timing shift in the burst pulse}

Next, we observed the timing shift in the burst pulse of the pulser. Figure 10(a) plots the pulse timing delay from the reference timing as a function of the pulse number from the 1 st to 48th pulse. Two problems emerge. First, we observed a large timing shift that changes in an exponential manner, $1-e^{-t / \tau}$, from the 1 st to 18 th pulse. Second, the pulse timing delay changes by about 800 ps for every third pulse. The amount of the timing change varies from pulser to pulser. The narrow band component inside the pulser seems to induce this timing shift, and the timing change every third pulse seems to derive from the interval change between 308 and $302.4 \mathrm{~ns}$. We introduced a trigger timing circuit to cancel the timing change every third pulse. Figure 10(b) shows the pulse timing after we adjusted the input trigger timing. A pulse timing in the range of $200 \mathrm{ps}$ from the 19th to 48th pulse is acceptable for multibunch beam extraction. The 1st to 18th pulses was discarded and not used for the beam kick.

\section{EXPERIMENTAL RESULTS}

Two strip-line kickers were installed as temporary replacements for the conventional pulsed magnet kicker, which was put offline.

Figure 11(a) shows the bunch current in the DR after three train injections. The bunch current was measured by a wall current monitor. Each train has ten bunches and each bunch has $5.6 \mathrm{~ns}$ spacing. Figure 11(b) shows the extracted multibunch beam signal. The blue line shows the bunch current measured by a current transformer located in the middle of the extraction line. The bunch spacing is $308 \mathrm{~ns}$ (302.4 ns for every third pulse) each. The intensity difference of each bunch depends on the bunch current in the DR.

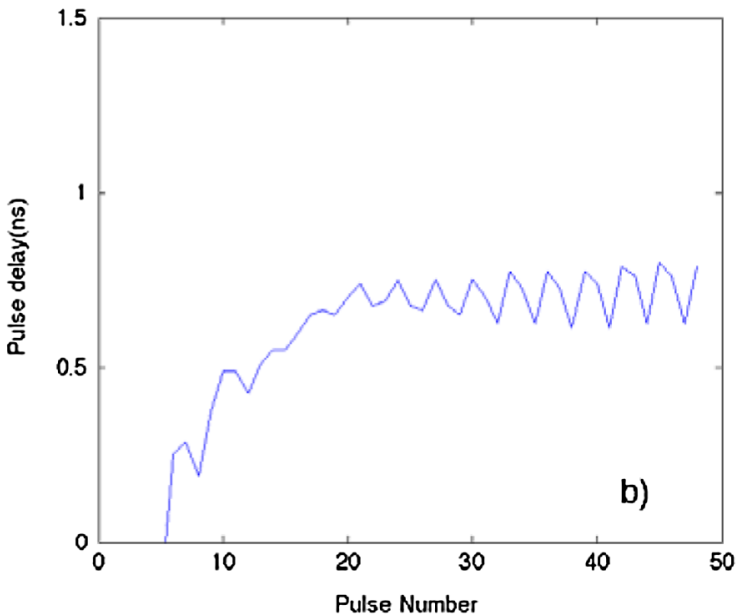

FIG. 10. (a) Time delay from the reference timing as a function of the pulse number. (b) Compensated time delay from the reference timing as a function of the pulse number. 

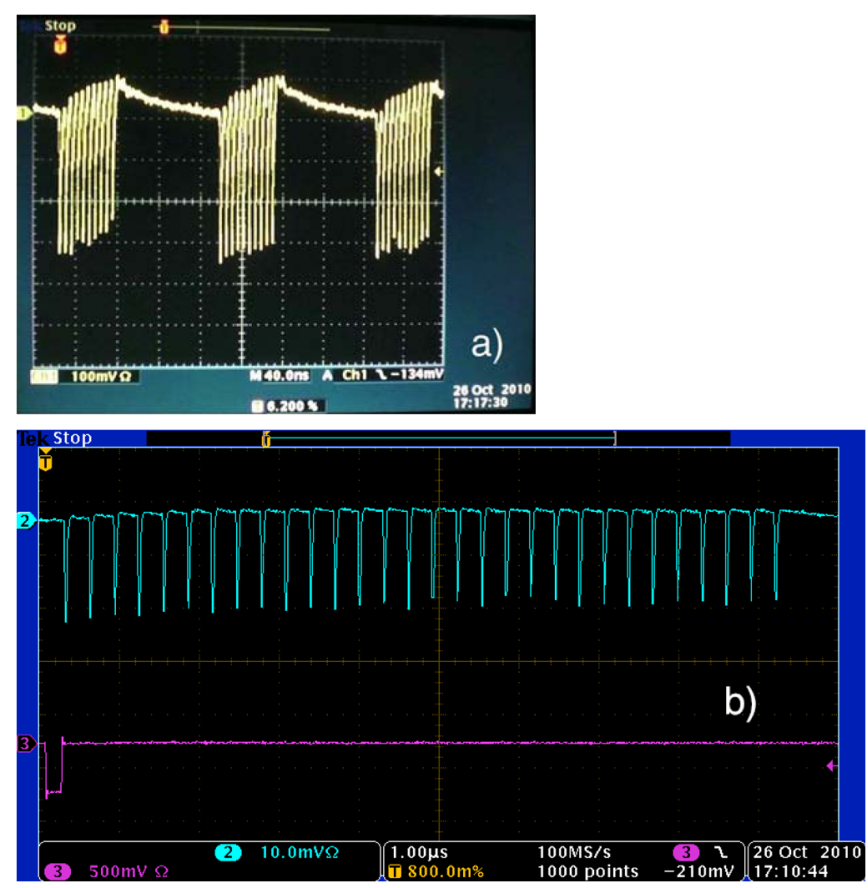

FIG. 11. (a) Bunch current in the DR: The stored beam consists of three trains and each train consists of ten bunches with $5.6 \mathrm{~ns}$ spacing. The vertical scale is $1 \mathrm{nC} / \mathrm{V}$. Vpeak is $400 \mathrm{mV}$ and the charge is $0.4 \mathrm{nC} /$ bunch. (b) Bunch current at the extraction line: A train consists of 30 bunches and each bunch has $308 \mathrm{~ns}$ (302.4 ns for every third pulse) spacing. The vertical scale is $22 \mathrm{nC} / \mathrm{V}$. Vpeak is $18 \mathrm{mV}$ and the charge is $0.4 \mathrm{nC} /$ bunch.

\section{A. Evaluation of the kick angle jitter for the single bunch}

We estimated the kick angle jitter for a single bunch from the orbit measurement of the extraction line. The kick

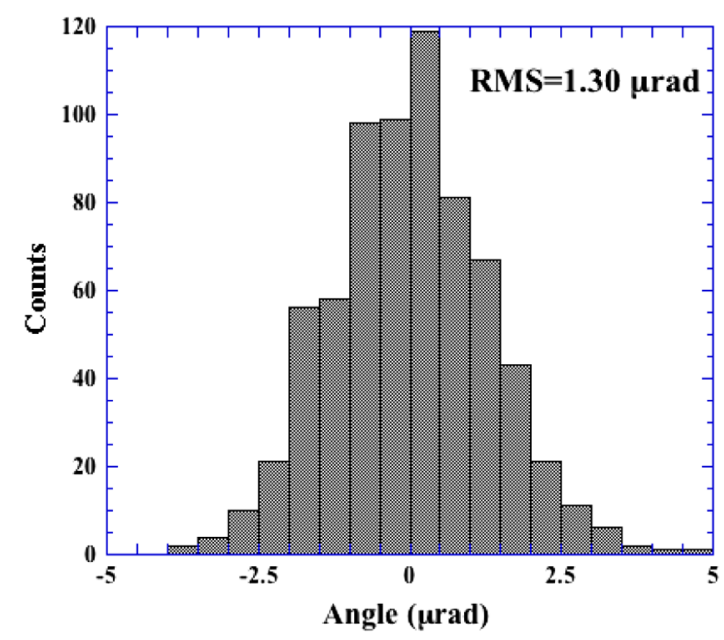

FIG. 12. The kick angle jitter distribution: Fit from the measured horizontal positions using 19 BPMs and the R12 of the design transfer matrix at each location estimates the angle distribution of the kicker.

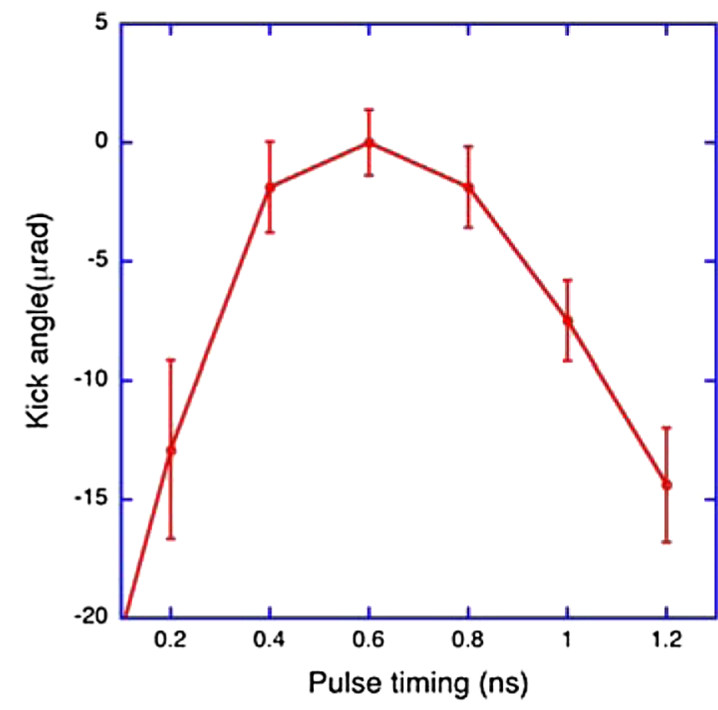

FIG. 13. The measured kick angle difference as a function of the kicker pulse timing.

angle is evaluated from the measured beam positions using 19 beam position monitors (BPMs) assuming designed beam line optics. Figure 12 shows the result of the kick angle distribution for 400 shots.

The measured angle jitter of the extracted beam was $1.3 \mu \mathrm{rad}$, which is $3.5 \times 10^{-4}$ of the kick angle. These results indicate that the strip-line kicker has a good stability comparable to that of the double kicker system of the pulse magnet [13]. As mentioned in Sec. II, the timing jitter of the kicker pulse dominates the kick angle jitter. The peak of the kick field had to be synchronized with the beam by adjusting the kick pulse timing in order to minimize the angle jitter; otherwise, the slope of the kick field would have increased the angle jitter. The measured timing jitter of the kicker pulse at the input trigger was less than $100 \mathrm{ps,} \mathrm{excluding} \mathrm{the} \mathrm{jitter}$ of the pulsers. The estimated minimum kick angle jitter when assuming a $200 \mathrm{ps}$ timing jitter was $3.3 \times 10^{-4}$, which was in agreement with the measurement.

Figure 13 shows the measured kick angle difference as a function of the kicker pulse timing. The kick angle is estimated from the beam position of one BPM. The error bar not only includes the kick angle jitter, but also the beam position jitter in the DR and the beam energy jitter in the DR. The shape of the kick angle difference near the peak is almost the same as the estimated kick field from the kicker pulse in Fig. 2(c).

\section{B. Evaluation of the kick angle and the kick angle jitter for the multibunch beam}

Next, we measured the kick angle and the angle jitter for the multibunch beam. Since we do not have a multibunch BPM system, we measured the beam position for a different kicker pulse by the following procedure: (1) extracting the beam for the different kicker pulse from the 1st to the 30th bunches; (2) using the single bunch BPM to 

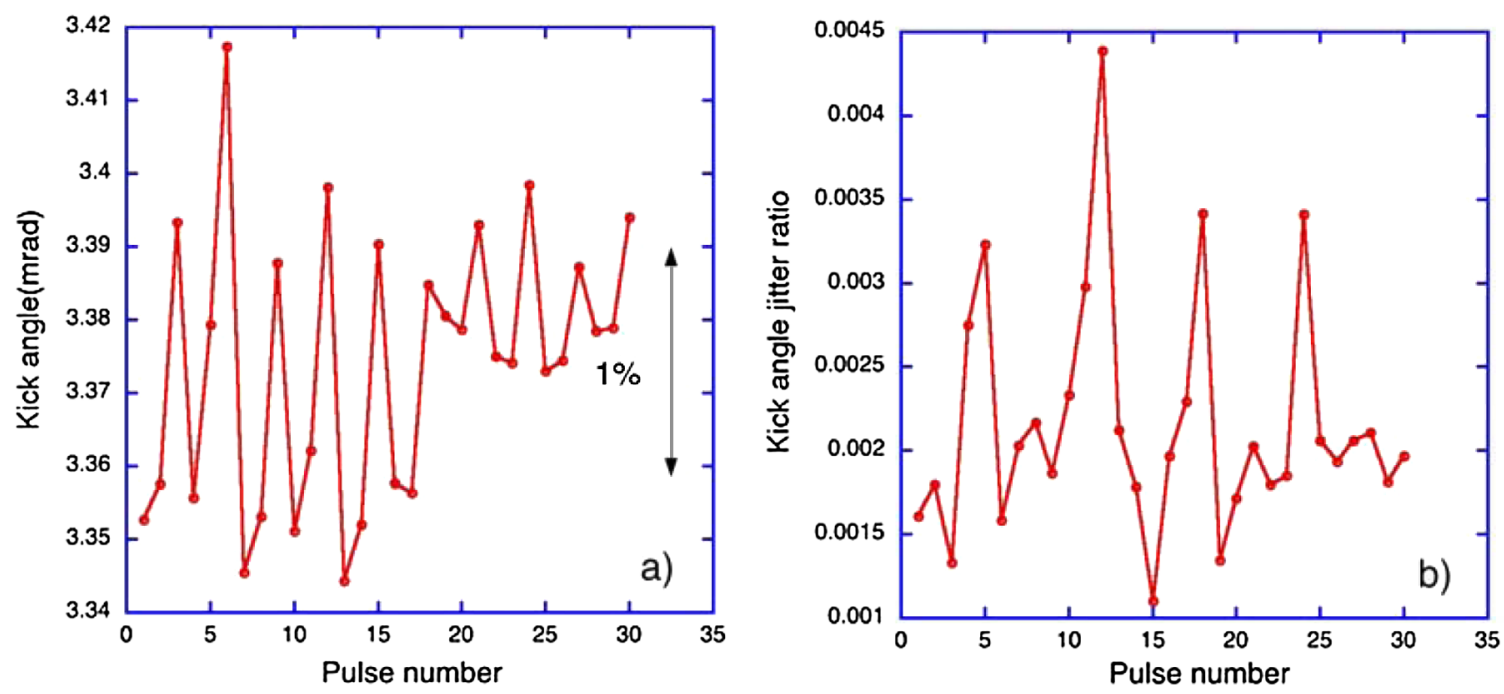

FIG. 14. (a) Kick angle as a function of the pulse number: The pulse-to-pulse variation of the kick angles of successive pulses is around $1 \%$. (b) Kick angle jitter ratio as a function of the pulse number: The jitter ratio for the kick angle was distributed from $1 \times 10^{-3}$ to $4 \times 10^{-3}$.

measure the beam position of the extracted beam by adjusting the gate timing of the BPM to the bunch timing. Five BPMs were used for the fitting in this analysis. Figure 14(a) shows the average of the kick angle of each kicker pulse. The pulse-to-pulse variation of the kick angles of successive pulses is around 1\%. Figure 14(b) shows the kick angle jitter ratio for each kicker pulse. The jitter ratio was distributed from $1 \times 10^{-3}$ to $4 \times 10^{-3}$. The results were worse than the single bunch result described in the previous subsection. The timing adjustment of the kicker pulse was insufficient in this measurement and the pulser characteristics mentioned in Sec. III E made it difficult to perform the fine adjustments for all of the kicker pulses. The feedback and/or feed-forward systems to minimize the kick angle and the angle jitter are also being developed for a long bunch train $[14,15]$. In the case of feed forward [15], Kalinin et al. outlined a system in which the bunch trajectory is measured with an upstream pair of BPMs and corrected with a pair of downstream fast kickers. The beam turnaround can allow signal processing and calculation of the correction.

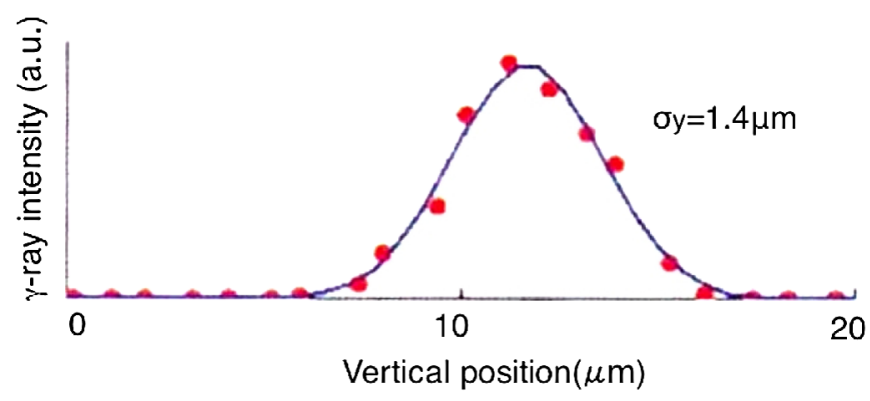

FIG. 15. The vertical beam size measurement with the wire scanner at the downstream of the focus point.

\section{The extracted beam profile}

The vertical beam size was measured with a wire scanner (named MS1IP) located immediately downstream of the focus point of the ATF2 beam line [16]. The measured size, $1.4 \mu \mathrm{m}$, was the measurement limit imposed by the size of the wire. Figure 15 shows the beam profile in the vertical direction as an intensity of the $\gamma$ ray. The same result was measured with the pulsed magnet kicker, which means that there was no apparent deterioration of the beam profile in the vertical direction.

\section{SUMMARY AND DISCUSSION}

The short bunch spacing multibunch beam in the DR was successfully extracted with long bunch spacing using the strip-line kicker. The beam was extracted in the same manner as for the ILC kicker. A pulse bump and auxiliary septum were used to compensate for the lack of a sufficient kick angle with the strip-line kicker. The angle jitter ratio of the single bunch beam was $3.5 \times 10^{-4}$, which was comparable to that of the double kicker system using pulsed magnets. The beam profile measurement of the wire scanner showed no apparent deterioration of the vertical beam profile. The timing control for the kicker pulse is key for the stable operation of the strip-line kicker. As the kick field of the strip-line kicker has no flattop, the system requires precise timing control at a subnanosecond level. This requires not only a stabilized trigger signal, but also pulser-topulser adjustment to accommodate different characteristics. The experiment could keep the kicker pulse timing in a range of 200 ps by using the timing control hardware.

To realize the ILC kicker requirements, we have to attain the following parameters: (1) bunch-by-bunch kicker amplitude stability $<0.07 \%$ and (2) droop of $1320-5120$ pulses burst $<0.07 \%$. To attain parameter (1), we consider 
improving the DC power supply of the pulser. The amplitude difference of the kicker pulse comes from the voltage change of the DC power supply. In addition, feed forward $[14,15]$ located at the turnaround section of ILC is expected to loosen the kick angle jitter tolerance. To attain parameter (2), we consider the use of a compensated DC power supply. The tested pulsers are FPG5-3000M fabricated by FID, each of which has a peak voltage of $5 \mathrm{kV}$, rate of $3 \mathrm{MHz}$. and 3000 pulses burst, which has a droop of $10 \%$. We fabricated another version, $2.5 \mathrm{kV}, 1 \mathrm{MHz}$ using BEHLKE HTS-50-80-UF FET switch, which has a droop of $0.5 \%$ in a burst of 500 pulses burst. This uses a large capacitance for the DC power supply. The droop can be reduced using a compensated DC power supply, which has electrical switches inside of the DC power supply to compensate the reduced voltage. The calculated droop was reduced from $0.5 \%$ to $0.006 \%$.

\section{ACKNOWLEDGMENTS}

The authors would like to express their gratitude to Professor S. Yamaguchi and Professor K. Yokoya for their encouragement and support. The authors would also like to thank the ATF collaborators for securing the machine time required for this experiment.

[1] ILC Global Design Effort and World Wide Study, "ILC Reference Design Report" [http://www.linearcollider.org/ about/Publications/Reference-Design-Report].
[2] T.S. Mattison et al., Part. Accel. 30, 115 (1990).

[3] T. Naito et al., in Proceedings of the 2007 Particle Accelerator Conference, Albuquerque, New Mexico (IEEE, New York, 2007), pp. 2772-2274.

[4] T. Naito et al., Nucl. Instrum. Methods Phys. Res., Sect. A 571, 599 (2007).

[5] B. I. Grishanov et al., Nucl. Instrum. Methods Phys. Res., Sect. A 396, 28 (1997).

[6] D. Alesini et al., Phys. Rev. ST Accel. Beams 13, 1110002 (2010).

[7] T. Naito et al., in Proceedings of the 23rd Particle Accelerator Conference, Vancouver, Canada, 2009 (IEEE, Piscataway, NJ, 2009), pp. 1620-1622.

[8] F. Hinode et al., KEK Internal Report No. 95-4, 1995.

[9] PEP-II Conceptual Design Report No. SLAC-418 (1993).

[10] OPERA-3D user guide, Vector Fields Limited, England.

[11] J.H. Billen et al., Los Alamos National Laboratory, Report No. LA-UR-96-1834.

[12] http://www.fidtechnology.com/products/fpg-nanosecond .html. The modified version (repetition rate and burst mode) is used in this report.

[13] T. Imai et al., KEK Report No. 2002-16, 2002.

[14] R. Apsimon et al., in Proceedings of IPAC10, 2010, Kyoto, Japan, pp. 2788-2790.

[15] A. Kalinin et al., in Proceedings of the 2007 Particle Accelerator Conference, Albuquerque, New Mexico (Ref. [3]), pp. 419-421.

[16] ATF2 Collaboration, KEK Report No. 2005-2/2005-9, 2005. 